\title{
BIOACUMULACIÓN DE NAFTALENO Y FLUORANTENO EN EL MOLUSCO BIVALVO Anadara tuberculosa (Sowerby, 1833)
}

\section{BIOACCUMULATION OF NAPHTHALENE AND FLUORANTHENE IN THE BIVALVE MOLLUSC Anadara tuberculosa (Sowerby, 1833)}

\author{
Mónica Zambrano ${ }^{1}$, Jorge Prada $^{2}$, Gustavo Arencibia ${ }^{3}$, Alexis Vidal $^{4}$
}

\begin{abstract}
${ }^{1}$ Bióloga Estudiante de Doctorado en Ciencias Biológicas. Centro de Investigaciones Marinas CIM. Universidad de La Habana. La Habana. Cuba. monyzambrano@yahoo.com; ${ }^{2}$ Biólogo. Área de Protección del Medio Marino. Centro de Investigaciones Oceanográficas e Hidrográficas del Pacífico CCCP, Tumaco Colombia. jepr83@hotmail.com; ${ }^{3}$ Doctor en Ciencias en el Uso, Manejo y Preservación de los Recursos. Centro de Investigaciones Pesqueras CIP. La Habana, Cuba. garen04@gmail.com; ${ }^{4}$ Doctor en Ciencias Farmacéuticas. Profesor Titular. Grupo de Farmacología-Toxicología, Facultad de Biología, Universidad de La Habana. La Habana, Cuba. alexis.vidal@infomed.sld.cu
\end{abstract}

Rev. U.D.C.A Act. \& Div. Cient. 15(2): 283 - 290, 2012

\section{RESUMEN}

Se realizó un seguimiento a la bioacumulación de naftaleno y de fluoranteno en el molusco bivalvo Anadara tuberculosa, mediante exposición de 60 organismos, a una concentración de $0,1 \mathrm{mg} / \mathrm{g}$ del compuesto de ensayo, en pruebas individuales para cada sustancia, por un periodo de 30 días. La máxima concentración de naftaleno acumulada por los organismos alcanzó los 3,42mg/g, luego de 15 días de exposición, mientras que el fluoranteno presentó niveles de $194,77 \mathrm{mg} / \mathrm{g}$, tras 5 días de prueba. En los organismos expuestos a naftaleno, se registraron variaciones en la concentración acumulada a lo largo de la prueba, contrario al comportamiento registrado en los organismos expuestos a fluoranteno, compuesto que presentó un incremento en concentración, durante la mayor parte del ensayo. Las diferencias en la cantidad acumulada de cada compuesto, se relacionan con las características fisicoquímicas de cada uno de ellos, contando el naftaleno con una menor estabilidad y mayor movilidad en los compartimentos ambientales frente al fluoranteno.

Palabras clave: Bioacumulación, hidrocarburos aromáticos policíclicos, naftaleno, fluoranteno, Anadara tuberculosa.

\section{SUMMARY}

The bioaccumulation of naphthalene and fluoranthene in the bivalve Anadara tuberculosa, was followed through the development of semistatic tests and exposure of organisms in individual tests for each compound at a concentration of $0.1 \mathrm{mg} / \mathrm{g}$ for a period of 30 days. The highest level of naphthalene accumulated by the organisms reached $3.42 \mathrm{mg} / \mathrm{g}$ after 15 days of exposure, while the lowest recorded concentration was presented after 20 days of test with 1.57 $\mathrm{mg} / \mathrm{g}$. For fluoranthene the initial concentration in organisms, when starting the trial was $5.88 \mathrm{mg} / \mathrm{g}$ and after exposure to the compound in the middle of the concentrations levels between $194.77 \mathrm{mg} / \mathrm{g}$ for 5 days of testing and $1011.30 \mathrm{mg} / \mathrm{g}$ for day 25 were registered. Fluctuations in terms of accumulation and purification of the compounds show a greater stability and incorporation of fluoranthene compared to naphthalene, which is related to the properties of each compound, especially those related to molecular weight and hydrophobicity.

Keywords: Bioaccumulation, polycyclic aromatic hydrocarbons, naphthalene, fluoranthene, Anadara tuberculosa.

\section{INTRODUCCIÓN}

La contaminación marina en las zonas costeras es un tema de interés mundial, debido a la gran cantidad de sustancias tóxicas que son transportadas por las actividades humanas (Vagi et al. 2005; Lors et al. 2009). Entre los contaminantes presentes en ambientes marinos, los hidrocarburos aromáticos policíclicos (HAP) constituyen uno de los principales problemas, debido a los efectos adversos que causan en la salud de los organismos (Zaghden et al. 2007). 
Algunos HAP son potenciales carcinógenos, mutágenos y teratógenos, tanto para los organismos acuáticos como para el hombre (Boscolo et al. 2007; Liu et al. 2009) y pueden afectar significativamente a los ecosistemas, influenciando las comunidades marinas planctónicas y bentónicas, así como organismos superiores, como peces y mamíferos (Pane et al. 2005), razón, por la cual, se encuentran en la lista de contaminantes prioritarios de la US EPA (Achten \& Hofmann, 2009; Rey-Salgueiro et al. 2009).

Los HAP, se caracterizan por su alta toxicidad, estabilidad en el medio ambiente y gran afinidad por los tejidos grasos, lo que favorece su bioacumulación y representan grandes riesgos, por las alteraciones que pueden generar; su incorporación por parte de los organismos y su capacidad de biomagnificación dan lugar a que se transporten, a través de la cadena trófica (Okay et al. 2000; Vagi et al. 2005).

Uno de los principales componentes en las fracciones solubles de los aceites petrogénicos en agua (Hansen et al. 2007) es el naftaleno, que constituye una de las fracciones más tóxicas del petróleo para la vida marina, por causar los mayores efectos narcóticos, entre todos los HAP (Vijayavel et al. 2004). Otro componente importante del contenido total de HAP en el ambiente es el fluoranteno (IARC, 1983), compuesto con un mayor potencial carcinogénico y otros efectos de tipo crónico, siendo un potente cocarcinógeno, con efectos mutagénicos (Irwin et al. 1997a).

Estos compuestos, al igual que otros contaminantes presentes en los ambientes acuáticos, son evaluados en las diferentes matrices ambientales, para lo cual, se pueden emplear organismos pertenecientes al grupo de los moluscos bivalvos; estos organismos se alimentan a través de filtración, proceso mediante el cual incorporan contaminantes en sus tejidos y son sésiles, lo que les hace fácilmente monitoreables.

Entre los moluscos presentes en el Pacífico colombiano, el molusco bivalvo Anadara tuberculosa es la especie de mayor importancia comercial (INCODER, 2007) y ha sido usada como organismo centinela en diferentes estudios de evaluación de la calidad ambiental en esta zona (Marrugo, 1993; Casanova, 1995; Sericano et al. 1995; Calero \& Zambrano, 1997; Velásquez \& Cortes, 1997).

Teniendo en cuenta los diversos eventos de contaminación registrados en la Bahía de Tumaco y dado que es en esta zona donde se realiza la mayor explotación de A. tuberculosa a lo largo del Pacífico colombiano, en el presente estudio, se evaluó la capacidad de bioacumulación presentada por organismos de esta especie, ante la exposición a diferentes concentraciones de naftaleno y de fluoranteno, compuestos que, debido a sus características, representan un riesgo importante para la salud de los organismos y para el hombre, como consumidor final de los mismos.

\section{MATERIALES Y MÉTODOS}

Los organismos de ensayo fueron adquiridos en el mercado local, procedentes de las zonas de manglar de la Bahía de Tumaco ( $1^{\circ} 53^{\prime} 10^{\prime \prime} \mathrm{N}$; $\left.78^{\circ} 39^{\prime} 50^{\prime \prime} \mathrm{W}\right)$; se trasladaron al Laboratorio de Bioensayos del Centro de Investigaciones Oceanográficas e Hidrográficas del Pacífico - Cccp, en donde fueron ubicados en tanques de $250 \mathrm{~L}$ de capacidad, con agua marina filtrada y aireación permanente, por un periodo de 72 horas.

Este periodo de aclimatación, se estableció teniendo en cuenta que para bioensayos en campo con moluscos bivalvos, debe tener una duración mínima de 48 horas, como periodo de depuración (APHA, AWWA, WEF, 2005). Transcurrido este periodo de tiempo, los organismos fueron distribuidos en doce acuarios de vidrio aforados a 20L para el inicio de las pruebas, colocándolos en número de diez en cada recipiente.

Previo inicio de las pruebas, se evaluó la estabilidad del naftaleno y del fluoranteno en el agua de disolución, bajo condiciones ambientales. Cada compuesto se diluyó en acetona y, luego, para cada uno de ellos, se preparó un recipiente de vidrio, con 3,0L de agua marina filtrada, al que se adicionaron $5,00 \mathrm{~mL}$ de una solución de $1,2 \mathrm{x}$ $10^{3} \mathrm{mg} / \mathrm{L}$, obteniendo una concentración de $2,0 \mathrm{mg} / \mathrm{L}$ de cada compuesto. Se tomaron $0,50 \mathrm{~L}$ del agua de cada recipiente, con periodicidad de seis horas, para determinar la concentración a lo largo de la prueba.

Posteriormente, se realizó una prueba de bioacumulación con cada compuesto, exponiendo ejemplares de $A$. tuberculosa a concentraciones de $0,1 \mathrm{mg} / \mathrm{L}$. Durante cada prueba, se expusieron un total de 60 organismos al compuesto de ensayo, 30 al agua de disolución (control) y 30 más a un control con adición de $10 \mathrm{~mL}$ de acetona (cantidad correspondiente a la proporcionada en solución con el compuesto de ensayo).

Durante las pruebas no se suministró aireación, pues esta acentúa la pérdida de los compuestos. Se realizaron recambios diarios del $100 \%$ del agua de disolución (cada 24 horas) en los recipientes de control (agua marina filtrada y solución de acetona) y en los recipientes de ensayo. El agua de recambio, se saturó con aire, con el fin de incrementar la concentración de oxígeno (OD).

Diariamente, se registraron los parámetros fisicoquímicos, salinidad, $\mathrm{pH}$, temperatura y oxígeno disuelto, empleando un 
equipo multiparámetros WTW Multi 340i. Con periodicidad de cinco días, se proporcionó alimento con el agua de recambio, suministrando diatomeas (Chaetoceros sp.), proporcionadas por el laboratorio de la empresa camaronera IDELPACIFICO S.A., con una densidad promedio de $3 \mathrm{x}$ $10^{6} \mathrm{cel} / \mathrm{mL}$, adicionando volúmenes equivalentes a $1 \mathrm{~L}$, en cada acuario.

Antes de iniciar cada bioensayo, se tomó una muestra de diez organismos, para determinar el contenido del compuesto que pudiesen presentar previo inicio de la prueba. La talla media registrada por los organismos de ensayo fue de 4,5 y $4,4 \mathrm{~cm}$ de longitud total (LT) para las pruebas con naftaleno y con fluoranteno, respectivamente.

Las muestras para determinar la concentración de los compuestos acumulados consistieron en tomar diez organismos, con periodicidad de cinco días, determinando, a la vez, el contenido de lípidos.

La determinación de lípidos, se realizó empleando el método de extracción directa Soxhlet, utilizando éter etílico como solvente y la de los compuestos de ensayo, siguiendo las metodologías descritas por Russell et al. (2002), para la determinación por cromatografía de gases, para lo cual, se empleó un cromatógrafo de gases (Agilent Technologies $6890 \mathrm{~N}$ ), acoplado a un detector selectivo de masas (Agilent Technologies - 5973N).

\section{RESULTADOS Y DISCUSIÓN}

La concentración inicial de naftaleno en el recipiente de ensayo equivalente a $2 \mathrm{mg} / \mathrm{g}$ descendió a $1,04 \mathrm{mg} / \mathrm{g}$, tras seis horas de exposición y a $0,5 \mathrm{mg} / \mathrm{g}$, transcurridas 12 horas, razón, por la cual, durante la prueba de bioacumulación, se realizó una adición del $50 \%$ de la concentración inicial, suministrada para reponer la cantidad de compuesto que se pierde por volatilización. Esta disminución en concentración obedece a que el naftaleno es un compuesto moderadamente volátil (Preuss et al. 2003).

El fluoranteno no registró disminución en la concentración, aun transcurridas 24 horas de ensayo, lo que evidencia una mayor estabilidad de este compuesto en el medio, asociada a un mayor peso molecular (PM).

Los niveles de naftaleno en los organismos al inicio del experimento fueron de $1,04 \mathrm{mg} / \mathrm{g}$ y durante la prueba los niveles del compuesto en los organismos variaron entre la concentración registrada, luego de 15 días de iniciada la prueba, con 3,42mg/g, como máximo nivel incorporado y la concentración presentada el día 20 , con $1,57 \mathrm{mg} / \mathrm{g}$, siendo este el nivel más bajo registrado durante el ensayo (Figura 1).

La incorporación de este compuesto presentó un comportamiento con ascensos y con descensos a lo largo de la prueba, ya que el naftaleno es el compuesto que presenta mayor solubilidad, lo que le hace más disponible, pero, además, es el compuesto más volátil y cuenta con la menor lipofilia

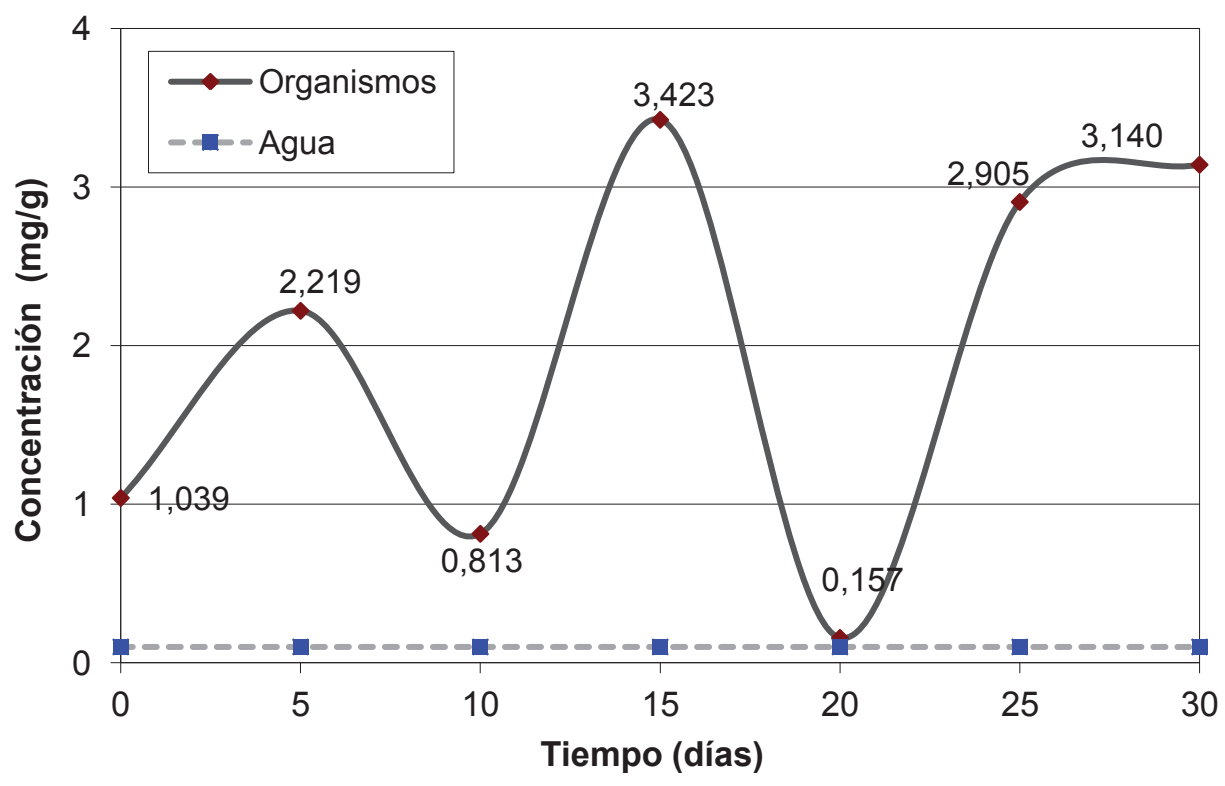

Figura 1. Comportamiento del naftaleno en Anadara tuberculosa durante la prueba de bioacumulación (mg/g - peso seco). 


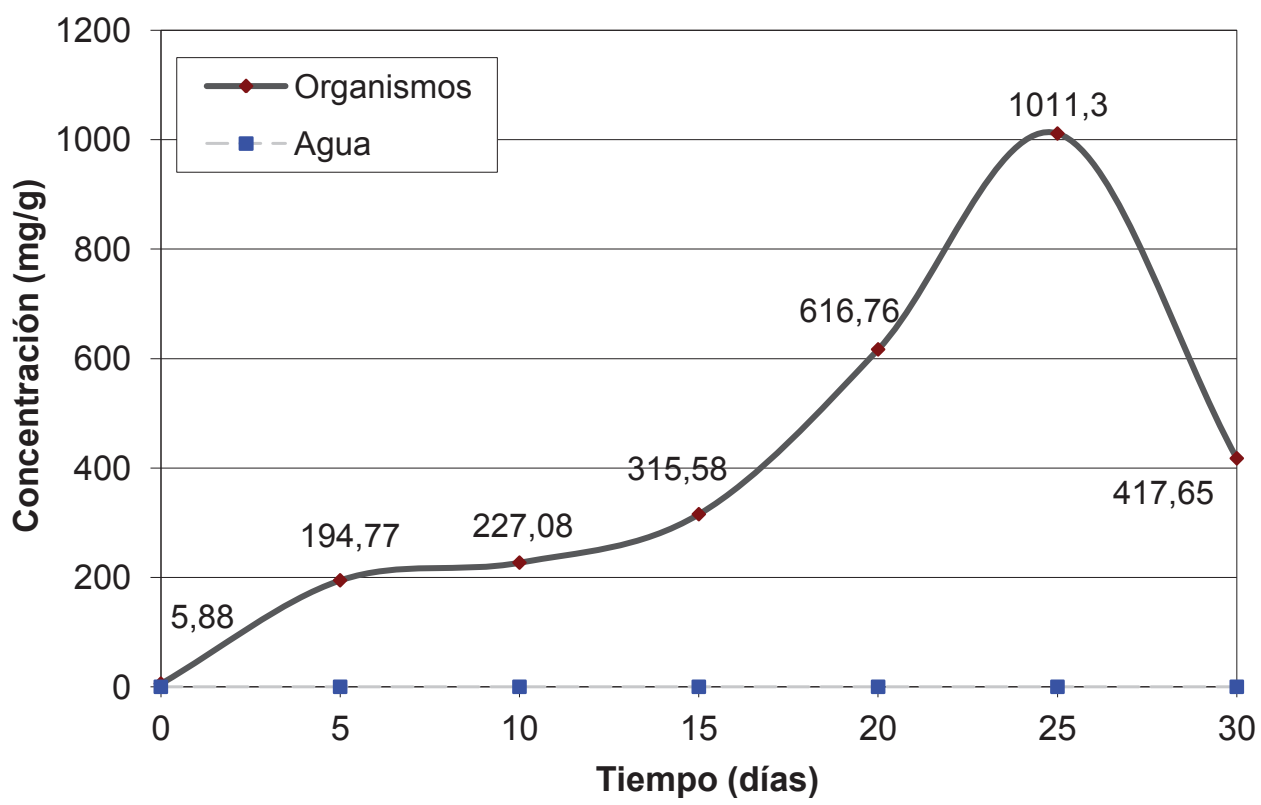

Figura 2. Comportamiento del fluoranteno en Anadara tuberculosa durante la prueba de bioacumulación (mg/g - peso seco).

dentro del grupo de los HAP (CCME, 2010), por lo que su retención por parte de los organismos es menos eficiente con respecto a los otros compuestos que poseen un mayor PM.

Este comportamiento cíclico se relaciona con el hecho que la bioconcentración del compuesto ocurre en un grado moderado; la depuración y el metabolismo proceden rápidamente en organismos acuáticos y, por esta razón, su impacto es considerado un problema de corto plazo (Irwin et al. 1997b).

El fluoranteno registró una concentración inicial de 5,88mg/g y, a lo largo de la prueba, las concentraciones incorporadas presentaron niveles entre los $194,77 \mathrm{mg} / \mathrm{g}$, para el quinto día de prueba y de $1011,30 \mathrm{mg} / \mathrm{g}$, para el día 25 . La tendencia de incorporación de este compuesto fue creciente hasta el día 25 de exposición, con un posterior descenso del $41,3 \%$, en la muestra correspondiente, al día 30 de ensayo (Figura 2).

Al igual que los HAP de alto peso molecular (4 - 6 anillos), el fluoranteno es más persistente que los compuestos más ligeros (Heath et al. 1993) y se acumula en mayor proporción con respecto a los mismos, tal y como se ha reportado en estudios realizados con otras especies de moluscos (Hellou et al. 1993; Krishnakumar et al. 1994; 1997).

Su incremento en concentración, se relaciona con los diferentes grados de lipofilia, de toxicidad y de persistencia de los HAP, que son más elevados cuanto mayor es el número de anillos de la molécula (Bocio et al. 2005); su posterior descenso sugiere un proceso eficiente de depuración tras alcanzar un determinado nivel de incorporación, comportamiento similar al presentado en estudios realizados con otros invertebrados expuestos a este compuesto (Landrum et al. 2002; Krishnakumar et al. 1997).

La captación, la acumulación y la persistencia depende de la relación entre la hidrofobicidad (relación entre el coeficiente de partición octanol/agua - $\operatorname{LogK}_{\text {ow }}$ ) y el contenido de lípidos (Belfroid et al. 1996), siendo el LogK $\mathrm{ow}_{\text {w }}$ para el naftaleno y el fluoranteno de 3,51 y 5,1, respectivamente (Peña et al. 2003), valores que se encuentran en el rango en el que se producen las mayores tasas de bioacumulación. A estas características, se suma como factor importante, la alta tasa de filtración que presentan los bivalvos, que para esta especie se estima en 50L/día (Fernández \& Brunker, 1977), siendo esta la principal ruta de incorporación.

Pese a las diferencias en cuanto a incorporación, ambos compuestos son bioacumulables, debido a sus características hidrofóbicas y lipofílicas, por lo que, una vez, ingeridos su eliminación metabólica se hace lenta y dificultosa, tendiendo a acumularse en órganos de almacenamiento, como los constituidos por tejidos grasos (Fernández \& Freire, 2005); sin embargo, son las características específicas de cada compuesto las que hacen que los niveles incorporados de naftaleno sean bastante inferiores con respecto al fluoranteno, pese a ser adicionados en concentraciones 
iguales, ya que su bajo PM le concede una mayor movilidad con respecto a otros HAP y, por ende, es retenido por cortos periodos de tiempo, tal y como se mencionó previamente.

Los ascensos y los descensos en la concentración de ambos compuestos constituyen un comportamiento usual en los organismos expuestos a contaminantes, pues estos los incorporan y los eliminan, aunque se mantenga la exposición a los mismos (Calero \& Zambrano, 1997; CSIC, 2003).

Los niveles de naftaleno y de fluoranteno incorporados por los organismos a lo largo de cada prueba fueron altos con respecto a la concentración proporcionada en el medio y evidencian la capacidad bioacumulativa de A. tuberculosa, lo que ratifica su importancia como organismo biomonitor y como una importante herramienta en la evaluación de contaminantes ambientales en el medio marino.

Contenido de lípidos: Durante ambas pruebas los organismos presentaron un comportamiento similar en cuanto a contenido lipídico, con ascensos y con descensos; estando influenciados por diferentes factores, entre ellos la presencia del compuesto.

Previo inicio de la prueba con naftaleno, el contenido lípidico registrado fue de $1,09 \%$ y de $1,43 \%$, para los organismos de la prueba realizada con fluoranteno. Los niveles máximos se registraron transcurridos 15 días de prueba, con 1,59\% y $1,77 \%$ y, los menores, a los de cinco días, con 0,58\% y 0,83\% para el naftaleno y fluoranteno, respectivamente (Figura 3).
Este descenso inicial en el contenido lípidico puede estar relacionado con una respuesta de los organismos ante las condiciones experimentales.

El mayor porcentaje de lípidos coincidió con el nivel más alto de naftaleno incorporado por los organismos, mientras que para el fluoranteno, se registró un ascenso importante en el contenido de lípidos, tras un significativo descenso en la concentración del compuesto.

El contenido de grasas constituye un aspecto fundamental en la retención de los HAP, pues son un importante reservorio de compuestos altamente liposolubles, por lo que el mismo se asocia con un mayor o menor grado de bioacumulación; sin embargo, el potencial de los tejidos para acumular estos compuestos esta en función de múltiples variables, como el estado nutricional, la concentración de lípidos, la duración de la exposición y la concentración en el medio (Riley et al. 1981).

El comportamiento de las grasas a lo largo de las pruebas no evidencia una relación con la concentración de los compuestos en los organismos, esto se debe, además, a que las movilizaciones de las reservas energéticas en respuesta a las condiciones ambientales y a que los contenidos de los diferentes sustratos energéticos pueden variar entre especies del mismo género; lo que ocurre en función de las necesidades metabólicas del organismo en un periodo dado (Acosta et al. 2010).

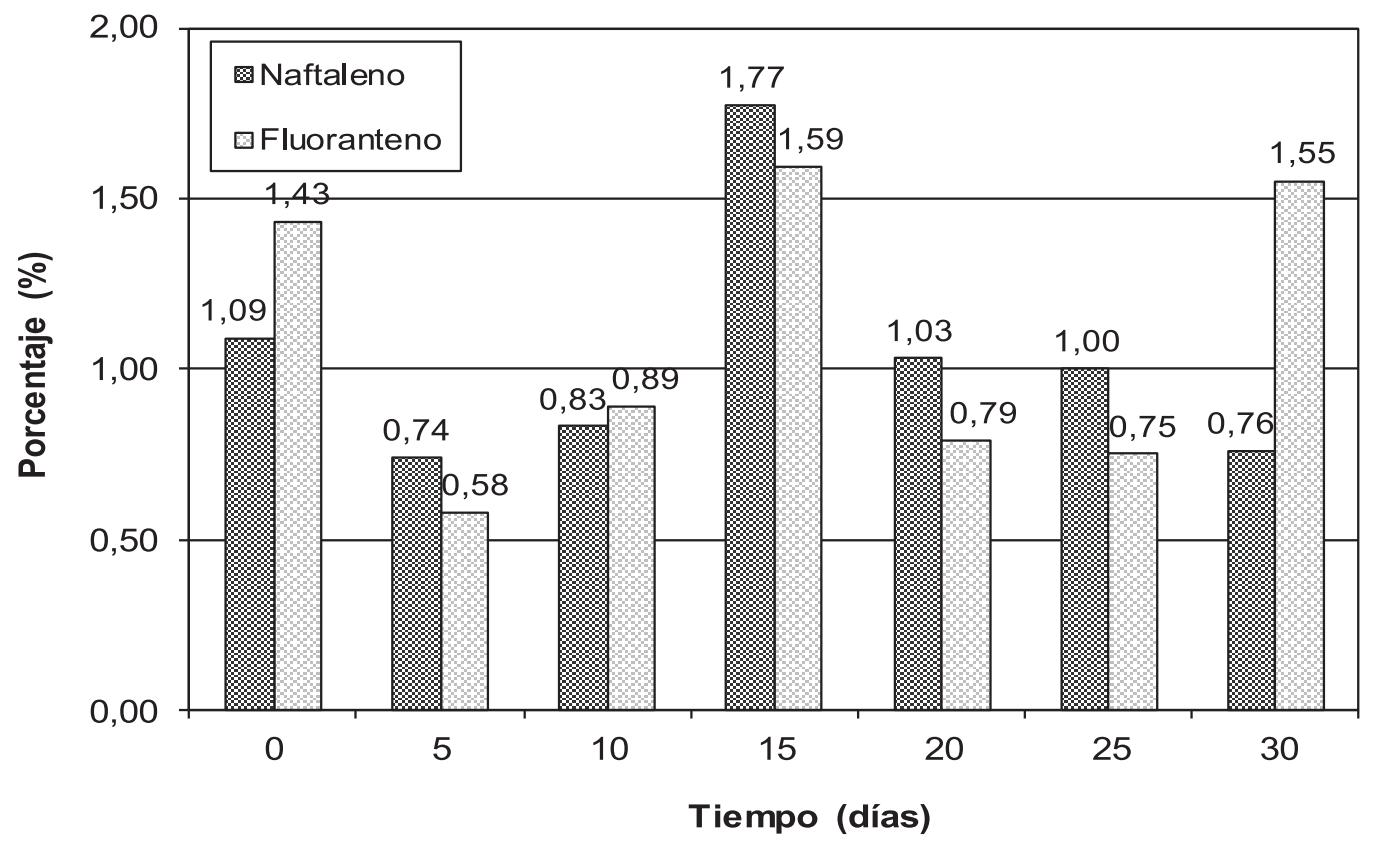

Figura 3. Comportamiento del contenido de grasas en Anadara tuberculosa durante las pruebas de bioacumulación. 
Parámetros fisicoquímicos: Los valores registrados durante las pruebas de bioacumulación fueron en promedio de $24,3^{\circ} \mathrm{C}$, para la temperatura; de 7,60 , para el $\mathrm{pH}$; de 30 ups, de salinidad y $4,7 \mathrm{mg} \mathrm{O}_{2} / \mathrm{L}$, de O.D.

Estos registros, se encuentran dentro de los rangos vitales conocidos para A. tuberculosa y otras especies del mismo género; en el caso específico del OD, esta especie tolera largos períodos de anoxia (Bayne, 1973), ya que las zonas estuarinas presentan cambios bruscos en los diferentes parámetros fisicoquímicos, causados por los cambios mareales (Prahl et al. 1990) y, en periodos de marea baja, la concentración de OD disminuye notablemente.

Los rangos de temperatura en los que se desarrolla la especie varían entre 26 y $35^{\circ} \mathrm{C}$ (Borrero, 1986), rangos en los que se encuentran los organismos dentro de la bahía; la salinidad es un factor importante en la distribución de la especie, con mayores densidades entre 10 y 34 ups, disminuyendo en sitios, cuya salinidad desciende a niveles que pueden afectar la reproducción y el crecimiento (Silva-Benavides \& BonillaCarrión, 2001).

La tolerancia de la especie a rangos amplios de parámetros fisicoquímicos y su adaptabilidad a condiciones de cautiverio, hacen de la misma un organismo adecuado en el desarrollo de este tipo de pruebas, permitiendo realizar evaluaciones del comportamiento y de los efectos adversos que generan determinados contaminantes al ingresar al ambiente marino.

Agradecimientos: Los autores agradecen a la Dirección del Centro de Investigaciones Oceanográficas e Hidrográficas del Pacífico - Cccp, por la financiación para el desarrollo del presente estudio; a los investigadores del Área de Protección del Medio Marino y al personal de apoyo civil y militar, por la colaboración logística durante el desarrollo del proyecto. Conflicto de intereses: El manuscrito fue preparado y revisado con la participación de todos los autores, quienes declaramos que no existe ningún conflicto de intereses que ponga en riesgo la validez de los resultados presentados. Financiación: Este estudio fue financiado por el Centro de Investigaciones Oceanográficas e Hidrográficas del Pacífico - Cccp de la Dirección General Marítima - DIMAR.

\section{BIBLIOGRAFÍA}

1. ACOSTA, V.; NATERA, Y.; LODEIROS, C.; FREITES, L.; VÁSQUEZ, A. 2010. Componentes bioquímicos de los tejidos de Perna perna y P. viridis (Lineo, 1758) (Bivalvia: Mytilidae), en relación al crecimiento en condiciones de cultivo suspendido. Lat. Am. J. Aquat. Res. (Chile). 38(1):37-46.
2. ACHTEN, C; HOFMANN, T. 2009. Native polycyclic aromatic hydrocarbons (PAH) in coals - a hardly recognized source of environmental contamination. Sci. Total Environ. 407:2461-2473.

3. APHA, AWWA, WEF. 2005. Standard Methods for the Examination of Water and Wastewater. 21st ed. American Public Health Association. Washington, DC. Part 8000 Toxicity. 8, 94-100.

4. BAYNE, B.L. 1973. The responses of three species of bivalve mollusk to declining oxygen tension at reduced salinity. Comp. Biochem. Phys. 45A:793806.

5. BELFROID, A.C.; SIJIM, D.T.H.M.; VAN GESTEL, C.A.M. 1996. Bioavailability and toxicokinetics of hydrophobic aromatic compounds in benthic and terrestrial invertebrates. Environ. Rev. (Canada). 4:276-299.

6. BORRERO, J.F. 1986. The collection of early juveniles of Anadara spp. as a potential source of seed for culturing mangrove cockles on the Pacific coast of Colombia. Aquaculture. 59:61-69.

7. BOCIO, A.; CASTELL, V.; FALCÓ, G.; GOSÁlBEZ, P.; RAMOS, J. 2005. Contaminants químics, estudi de dieta total a Catalunya. Agència Catalana de Seguretat Alimentària. Generalitat de Catalunya. Generalitat de Catalunya. Barcelona (España). 143p.

8. BOSCOLO, R., CACCIATORE, F.; GIOVANARDI, O. 2007. Polycyclic aromatic hydrocarbons (PAHs) in transplanted Manila clams (Tapes philippinarum) from the Lagoon of Venice as assessed by PAHs/shell weight index: a preliminary study. Mar. Pollut. Bull. 55:485-493.

9. CALERO, L; ZAMBRANO, M. 1997. Bioacumulación de hidrocarburos aromáticos del petróleo en un molusco bivalvo Anadara tuberculosa. Bol. Cient. CCCP. (Colombia). 6:131-137.

10. CASANOVA, R. 1995. Estudio de la contaminación marina por compuestos organoclorados en el Pacífico colombiano. Bol. Cient. CCCP (Colombia). 5:146-160.

11. CCME (Canadian Council of Ministers of the Environment). 2010. Canadian Soil Quality Guidelines for Carcinogenic and Other Polycyclic Aromatic Hydrocarbons (Environmental and 
Human Health Effects). Scientific Criteria Document (revised). Quebec (Canada). 216p.

12. CSIC (Consejo Superior de Investigaciones Científicas). 2003. Impacto de un vertido de petróleo sobre los organismos marinos. Algunas lecciones del vertido del Aegean sea. Informe técnico No. 15. (España). Disponible desde Internet en: http://csicprestige. iim.csic.es/informes/info15.pdf (con acceso 03/04/2011).

13. FERNÁNDEZ, B.; BRUNKER, T. 1977. Estudio bacteriológico de bivalvos del Golfo de Nicoya, Costa Rica (I Parte). Rev. Biol. Trop. (Costa Rica). 25:101107.

14. FERNÁNDEZ, L.; FREIRE, J. 2005. Relaciones entre la salud de los ecosistemas marinos y la seguridad alimentaria: La marea negra del Prestige como un caso de estudio. (España). Disponible desde Internet en: http://otvm.uvigo.es/investigacion/ informes/documentos/archivos/Salud_ecosistemas seguridad_alimentaria.pdf (con acceso 04/06/2011).

15. HANSEN, B.H.; ALTIN, D.H.; VANG, SH.; NORDTUG, T.; OLSEN, A.J. 2007. Effect of naphthalene o gene transcription in Calanus finmarchinus (Crustacea, Copepoda). Aquatic Toxicol. 86:157-165.

16. HEATH, J.S.; KOBLIS, K.; SAGER, S.; DAY, C. 1993. Risk assessment for total petroleum hydrocarbons. In: Irwin, R.J.; VanMouwerik, M.; Stevens, L.; Seese, M.D.; Basham, W. (eds). Environmental Contaminants Encyclopedia Fluoranthene Entry. National Park Service, Water Resources Division, Fort Collins, Colorado. (Estados Unidos). Disponible desde internet en: http://www.nature. nps.gov/hazardssafety/toxic/fluorant.pdf (con acceso 03/04/2011).

17. HELLOU, J.; UPSHALL, C.; PAYNE, J.F.; NAIDU, S.; PARANJAPE, M.A. 1993. Total unsaturated compounds and polycyclic aromatic hydrocarbons in mollusks collected from waters around Newfoundland. Arch. Environ. Contam. Toxicol. 24(2):249-257.

18. IARC. 1983. Polynuclear Aromatic Compounds: Part 1, Chemical, environmental and experimental data. IARC Monographs on the Evaluation of Carcinogenic Risks to Humans. WHO. Vol. 32. p.95-431, (Francia). Last updated: 17 April 1998.
19. INCODER. 2007. Sistema de información sectorial pesquero. En: Estado actual del cultivo y manejo de moluscos bivalvos y su proyección futura: factores que afectan su sustentabilidad en América Latina. (A. Lovatelli, A. Farias e I. Uriarte eds.). Taller Técnico Regional de la FAO. 20-24 de agosto de 2007, Puerto Montt, Chile. FAO Actas de Pesca y Acuicultura. No. 12. Roma (Italia), FAO. p.115-128.

20. IRWIN, R.J.; VANMOUWERIK, M.; STEVENS, L.; SEESE, M.D.; BASHAM, W. 1997a. Environmental Contaminants Encyclopedia Fluoranthene Entry. National Park Service, Water Resources Division, Fort Collins, Colorado. (Estados Unidos). Disponible desde Internet en: http:/www.nature. nps.gov/hazardssafety/toxic/fluorant.pdf (con acceso 07/05/2011).

21. IRWIN, R.J.; VANMOUWERIK, M.; STEVENS, L.; SEESE, M.D.; BASHAM, W. 1997b. Environmental Contaminants Encyclopedia Naphthalene Entry. National Park Service, Water Resources Division, Fort Collins, Colorado. (Estados Unidos). Disponible desde internet en: http://www.nature.nps.gov/ hazardssafety/toxic/naphthal.pdf (con acceso 07/05/2011).

22. KRISHNAKUMAR, P.K.; CASILLAS, E.; VARANASI, U. 1994. Effects of environmental contaminants on the health of Mytilus edulis from Puget Sound, Washington, USA. I. Cytochemical measures of lysosomal responses in the digestive cells using automatic image analysis. Mar. Ecol. Prog. Ser. 106:249-261.

23. KRISHNAKUMAR, P.K.; CASILLAS, E.; VARANASI, U. 1997. Cytochemical reponses in the digestive tissue of Mytilus edulis complex exposed to microencapsulated PAH or PCBs. Comp. Biochem. Physiol. 118:11-18.

24. LANDRUM, P.F.; GEDEON, M.L.; BURTON, G.A.; GREENBERG, M.S.; ROWLAND, C.D. 2002. Biological Responses of Lumbriculus variegatus Exposed to Fluoranthene-Spiked Sediment. Arch. Environ. Contam. Toxicol. 42:292-302.

25. LIU, Y.; CHEN, L.; HUANG, Q.H.; LI, W.Y.; TANG, Y.J.; ZHAO, J.F. 2009. Source apportionment of polycyclic aromatic hydrocarbons (PAH) in surface sediments of the Huangpu River, Shangai, China. Sci. Total Environm. 407:2931-2938.

26. LORS, C.; PERIE, F.; GRAND, C.; DAMIDOT, D. 
2009. Benefits of ecotoxicological bioassays in the evaluation of a field biotreatment of PAHs polluted soil. Global NEST J. 11(3):251-259.

27. MARRUGO, A.J. 1993. Estudio de la contaminación marina por hidrocarburos en el Pacífico Colombiano - Fase III. Bol. Cient. CCCP. (Colombia). 4:47-60.

28. OKAY, O.S.; DONKIN, P.; PETERS, L.D.; LIVINGSTONE, D.R. 2000. The role of algae (Isochrysis galbana) enrichment on the bioaccumulation of benzo[a] pyrene and its effects on the blue mussel Mytilus edulis, Environ. Pollut. 110:103-113.

29 PANE, L.; BOCCARDO, S.; BONFIGLIOLI, F.; MARIOTTINI, G.L.; PRIANO, F.; CONIO, O. 2005. Polycyclic aromatic hydrocarbons in water, seston and copepods in a harbour area in the Western Mediterranean (Ligurian Sea). Mar. Ecol. 26:89-99.

30. PEÑA, A.; MORALES, J.; LABASTIDA, A.; CAPELLA, S. 2003. Extracción en Fase Sólida como una Alternativa para el Procedimiento de Limpieza en la Determinación de Hidrocarburos Aromáticos Policíclicos por Cromatografía de Gases. Rev. Int. Contam. Ambient. (México). 19(1):13-23.

31. PRAHL, H. von; CANTERA, J.R.; CONTRERAS, R. 1990. Manglares y hombres del Pacífico colombiano. Fondo FEN. Bogotá (Colombia). 193p.

32. PREUSS, R.; ANGERER, J.; DREXLER, H. 2003. Naphthalene an environmental and occupational toxicant. Int. Arch. Occup. Environ. Health. 76(8):556-576.

33. REY-SALGUEIRO, L.; MARTÍNEZ-CARBALLO, E.; GARCÍA-FALCÓN, M.; SIMAL-GÁNDARA, J. 2009. Survey of polycyclic aromatic hydrocarbons in canned bivalves and investigation of their potential sources. Food. Res. Int. 42:983-988.

34. RILEY, R.T.; MIX, M.C.; SCHAFFER, R.L.; BUNTING, D.L. 1981. Uptake and accumulation of naphthalene by the oyster Ostrea edulis, in a flow-through system. Mar. Biol. 61(4):267-276.

35. RUSSELL, M.L.; GOTH-GOLDSTEIN, R.; APTE, M.G.; FISK, W.J. 2002. Analytical Method for the determination of Individual (N-alkanes and
Isoprenoids) and Total Petroleum Hydrocarbons (TPH), Polycyclic Aromatic Hydrocarbons (PAHs) and Biomarker Triterpanes and Steranes in Oil and Oil-spill-related. Environmental Samples (Water, Particulates and Soils/Sediment): Berkeley (CA): Lawrence Berkeley National Laboratory, Environmental Energy Technologies Division; 2002 Jan. Report No.: LBNL49574. Contract No.: DEAC0376SF00098. Sponsored by the Department of Energy. Ottawa. ON, K1A OH3.

36. SERICANO, J.L.; WADE, T.L.; JACKSON, T.J.; BROOKS, J. M.; TRIPP, B.W.; FARRINGTON, J.W.; MEE, L.D.; READMAN, J.W.; VILLENEUVE, J.P.; GOLDBERGS, E.D. 1995. Trace organic contamination in the Americas: An Overview of the US National Status \& Trends and the International "Mussel Watch" Programmes. Mar. Pollut. Bull. 31(4-12):214-225.

37. SILVA BENAVIDES, A.M.; BONILLA CARRIÓN, R. 2001. Abundancia y morfometría de Anadara tuberculosa y A. similis (Mollusca: Bivalvia) en el Manglar de Purruja, Golfo Dulce, Costa Rica. Rev. Biol. Trop. (Costa Rica). 49(2):315-320.

38. VAGI, M.C.; KOSTOPOULOU, M.N.; PETSAS, A.S.; LALOUSI, M.E.; RASOULI, C.H.; LEKKAS, T.D. 2005. Toxicity of organophoshorous pesticides to the marine alga Tetraselmis suecica. Global NEST J. 7(2):222-227.

39. VELÁSQUEZ, O.; CORTES, L.M. 1997. Estudio y evaluación de metales traza $(\mathrm{Pb}, \mathrm{Cr}, \mathrm{Cu}, \mathrm{Cd}$ y Hg) en aguas, sedimentos y organismos marinos de la bahía de Buenaventura. Bol. Cient. CCCP (Colombia). 6:57-61.

40. VIJAYAVEL, K.; GOMATHI, R.D.; DURGABHAVANI, K.; BALASUBRAMANIAN, M.P. 2004. Sublethal effect of naphthalene on lipid peroxidation and antioxidant status in the edible marine crab Scylla serrata. Mar. Pollut. Bull. 48:429-433.

41. ZAGHDEN, H.; KALLEL, M.; ELLEUCH, B.; OUDOT, J.; SALIOT, A. 2007. Sources and distribution of aliphatic and polyaromatic hydrocarbons in sediments of Sfax, Tunisia. Mediterranean Sea, Mar. Chem. 105(1-2):70-89.

Recibido: Octubre 11 de 2011

Aceptado: Junio 15 de 2012 